\title{
Article \\ Supersymmetric Partners of the One-Dimensional Infinite Square Well Hamiltonian
}

\author{
Manuel Gadella $^{1}\left(\mathbb{D}\right.$, José Hernández-Muñoz ${ }^{2}\left(\mathbb{D}\right.$, Luis Miguel Nieto ${ }^{1, *(\mathbb{D})}$ and Carlos San Millán ${ }^{1}(\mathbb{D})$ \\ 1 Departamento de Física Teórica, Atómica y Óptica and IMUVA, Universidad de Valladolid, \\ 47011 Valladolid, Spain; gadella@fta.uva.es (M.G.); carlos.san-millan@alumnos.uva.es (C.S.M.) \\ 2 Departamento de Física Teórica de la Materia Condensada, IFIMAC Condensed Matter Physics Center, \\ Universidad Autónoma de Madrid, 28049 Madrid, Spain; jose.hernandezm@uam.es \\ * Correspondence: luismiguel.nieto.calzada@uva.es; Tel.: +34-983-423754
}

Citation: Gadella, M.

Hernández-Muñoz, J.; Nieto, L.M.; San Millán, C. Supersymmetric Partners of the One-Dimensional Infinite Square Well Hamiltonian Symmetry 2021, 13, 350. https:// doi.org/10.3390/sym 13020350

Academic Editor: Georg Junker

Received: 1 February 2021

Accepted: 17 February 2021

Published: 21 February 2021

Publisher's Note: MDPI stays neutral with regard to jurisdictional claims in published maps and institutional affiliations.

Copyright: (c) 2021 by the authors. Licensee MDPI, Basel, Switzerland. This article is an open access article distributed under the terms and conditions of the Creative Commons Attribution (CC BY) license (https:// creativecommons.org/licenses/by/ $4.0 /)$.

\begin{abstract}
We find supersymmetric partners of a family of self-adjoint operators which are self-adjoint extensions of the differential operator $-d^{2} / d x^{2}$ on $L^{2}[-a, a], a>0$, that is, the one dimensional infinite square well. First of all, we classify these self-adjoint extensions in terms of several choices of the parameters determining each of the extensions. There are essentially two big groups of extensions. In one, the ground state has strictly positive energy. On the other, either the ground state has zero or negative energy. In the present paper, we show that each of the extensions belonging to the first group (energy of ground state strictly positive) has an infinite sequence of supersymmetric partners, such that the $\ell$-th order partner differs in one energy level from both the $(\ell-1)$-th and the $(\ell+1)$-th order partners. In general, the eigenvalues for each of the self-adjoint extensions of $-d^{2} / d x^{2}$ come from a transcendental equation and are all infinite. For the case under our study, we determine the eigenvalues, which are also infinite, all the extensions have a purely discrete spectrum, and their respective eigenfunctions for all of its $\ell$-th supersymmetric partners of each extension.
\end{abstract}

Keywords: supersymmetric quantum mechanics; self-adjoint extensions; infinite square well; contact potentials

\section{Introduction}

The study of one dimensional models in quantum mechanics is useful in order to gain a better understanding of the properties of quantum systems. In particular, the construction of supersymmetric (SUSY) partners of given potentials allow for an analysis of one dimensional Hamiltonians that often keep similarities with the original ones. Many studies have been done in this field and a brief account of references [1-14] only covers a small part of all previous work.

In the present paper, we intend to investigate the properties of the SUSY partners of the self-adjoint determinations of the operator $-d^{2} / d x^{2}$ on $L^{2}[-a, a], a>0$ and finite, with appropriate boundary conditions at the points $-a$ and $a$. Note that this problem is closely related to the problem of the definition of the "free" Hamiltonian on the one dimensional infinite square well potential.

From our point of view, SUSY quantum mechanics is a method that pursues the identification of the class of Hamiltonians for which their spectral problem can be algebraically solved. Traditionally, people have investigated SUSY partners of well studied exactly solvable Hamiltonians that give rise to other Hamiltonians for which the spectrum coincides with the spectrum of the original Hamiltonian except for one eigenvalue. In addition, there are several examples in which one original Hamiltonian produces an infinite chain of Hamiltonians, the first element of the chain being its SUSY partner and each of the others is a partner of the previous and the next one. Here, we explore the possibility of obtaining the whole chain of partners corresponding to self-adjoint extensions of a symmetric one dimensional Hamiltonian with equal deficiency indices. Since in our case, the variety of 
self-adjoint extensions is quite wide, depending on four real parameters, we have expected to find interesting new results in the field as it happened to be.

The analysis of these self-adjoint extension has been done in [15]. The task of computing the SUSY partners of all the self-adjoint determinations (also called extensions) of $-d^{2} / d x^{2}$ on $L^{2}[-a, a]$, their spectra and their wave functions is not trivial, although can be carried out systematically.

Although the idea of self-adjoint extensions of symmetric (or Hermitian) operators on (infinite dimensional) Hilbert spaces is not yet very popular among physicists, it is, however, possible to find recent papers on the topic [16-24]. Standard quantum mechanics textbooks refer to the one dimensional infinite square well potential or the harmonic oscillator as if they were described by a unique self-adjoint Hamiltonian, which produces a neatly calculable spectrum. The mathematical reality is much more complex and may give many more possibilities for the study of quantum mechanics systems. Let us briefly address to this problem, for which a more thorough presentation can be found in mathematical textbooks [25] as well as papers addressed to the Physics community [15].

Concerning terminology, an operator, $A$, on a infinitely dimensional separable Hilbert space (the Hilbert space must be infinite dimensional, since otherwise all operators are continuous and defined on the whole space. In such a case, this argumentation does not make sense. A separable Hilbert space is one with a countable orthonormal basis, which is always the case in ordinary quantum mechanics) $\mathcal{H}$ is symmetric, or equivalently Hermitian if for any pair of vectors $\varphi, \psi \in \mathcal{D}(A)$, where $\mathcal{D}(A)$ is the domain of $A$, which must be densely defined, one has that $\langle A \varphi \mid \psi\rangle=\langle\varphi \mid A \psi\rangle$, where $\langle-\mid-\rangle$ denotes the scalar product on $\mathcal{H}$. This means that the adjoint, $A^{\dagger}$, of $A$ extends $A, A \prec A^{\dagger}$ (i.e., $\mathcal{D}(A) \subset \mathcal{D}\left(A^{\dagger}\right)$ and $A \psi=A^{\dagger} \psi$, for all $\left.\psi \in \mathcal{D}(A)\right)$. The deficiency indices are $n_{ \pm}:=\operatorname{dim} \operatorname{Ran}\left(A^{\dagger} \pm i I\right)$, where $\operatorname{Ran}(B)$ is the range (image space) of the operator $B$ and $I$ is the identity operator. A symmetric (or Hermitian) operator has self-adjoint determinations (or extensions) if and only if $n_{+}=n_{-}$[25]. If $n_{+}=n_{-}=0$, this extension is unique. On the other hand, if $n_{+}=n_{-} \neq 0$, the number of extensions is infinite and, in the case of Hilbert spaces of functions, they usually can be determined by some matching or boundary conditions that the functions in the domain of the extensions should fulfill at some points [15,25-27].

Self-adjoint determinations of the operator $-d^{2} / d x^{2}$ defined on functions supporting whatever interval, $\mathcal{K}$, in the real line $\mathbb{R}$ are used to define the so call contact potentials [26,28-31]. These are perturbations of the "free operator" $H_{0}=-d^{2} / d x^{2}$, which are supported on a single point $x_{0} \in \mathcal{K}$. Typical examples of contact potentials are the Dirac delta $\delta\left(x-x_{0}\right)$ or its derivative $\delta^{\prime}\left(x-x_{0}\right)$, which define Hamiltonians of the type $H_{0}+\delta\left(x-x_{0}\right)$ or $H_{0}+\delta^{\prime}\left(x-x_{0}\right)$ as well defined self-adjoint operators on the Hilbert space $L^{2}(\mathcal{K})$ [27]. These types of perturbations may serve as a good and tractable approximation for a very localized spatial perturbation and are defined via matching conditions that must satisfy the functions on the domain of the operator at $x_{0}$. Concerning the operator $-d^{2} / d x^{2}$ on $L^{2}[-a, a]$, some relations have been found among the boundary conditions at the borders $-a$ and $a$ and matching conditions defining a $\delta$ or $\delta^{\prime}$ perturbation at the origin [32,33].

A comment is of relevance here. Let us consider the subspace, $\mathcal{D}_{0}$, of all twice differentiable square integrable functions, $\varphi(x)$, in the interval $[-a, a]$, with second derivative in $L^{2}[-a, a]$, verifying the boundary conditions $\varphi(-a)=\varphi(a)=\varphi^{\prime}(-a)=\varphi^{\prime}(a)=0$, and a differential operator of the form

$$
D=-\frac{d^{2}}{d x^{2}}+p_{1}(x) \frac{d}{d x}+p_{2}(x),
$$

where $p_{1}(x)$ and $p_{2}(x)$ are continuous real functions (with $p_{1}(x)$ differentiable) on $[-a, a]$. Then $D$ is Hermitian on $\mathcal{D}_{0}$ with deficiency indices $(2,2)$. It has been proven in [34] (vol. 2, p. 90) that all self-adjoint extensions of $D$ have a purely discrete spectrum. This is precisely the case of all the self-adjoint determinations of $-d^{2} / d x^{2}$ under our study [15] These self-adjoint extensions are characterized by a set of four real parameters, so that one 
particular choice of these parameters gives a unique self-adjoint determination of $-d^{2} / d x^{2}$ on $L^{2}[-a, a]$ and vice-versa. Although this is much less known, a similar situation emerges in the study of the one dimensional harmonic oscillator [35].

The present article intends in the first place, to complete as far as possible, the classification of the self-adjoint extensions of $-d^{2} / d x^{2}$ on $L^{2}[-a, a]$ given by [15]. Once this task has been done, we intend to obtain the whole chain of SUSY partners of each of the selfadjoint extensions using standard methods already developed in the theory [1]. This kind of supersymmetry intends to construct a series of potentials (in our case one-dimensional), with an energy spectrum closely related and that can be obtained from the spectrum of the original potential. Thus, being given one of our original self-adjoint extensions and being known the solution of the spectral problem, we should be able to obtain an infinite sequence of Hamiltonians such that their spectra coincides with the spectra of the previous one except for one eigenvalue, and hence from the original one except for a finite number of energy levels. We must add that all self-adjoint extensions of $-d^{2} / d x^{2}$ on $L^{2}[-a, a]$ have a purely discreet spectrum with an infinite number of energy levels.

The ground state for each of these extensions either has a strictly positive, zero or negative energy. Obviously, in the latter case, this fact comes from extensions which are not definitely positive. This is somehow paradoxical, due to the form of the original operator, which is $-d^{2} / d x^{2}$. This paradox is solved in [15]. For those extensions with a ground state with strictly positive energy, we have constructed the whole sequence of its SUSY partners and have given the eigenvalues and eigenfunctions for these partners. As mentioned earlier, the set of eigenvalues for each partner comes from the set of eigenvalues of the extension from which we construct the sequence of partners.

The general formalism can also be applied to obtain a sequence of Hamiltonians when the ground state of the original self-adjoint extension of $-d^{2} / d x^{2}$ on $L^{2}[-a, a]$ has zero or negative energy. In this case, partner Hamiltonians may be very different from the original one in the sense that they may have a finite number of eigenvalues or simply no eigenvalues. This is due to the presence of nodes in the wave function of the ground state. Nevertheless, these partners may be obtained and classified, although this discussion is left for a future publication.

This paper is organized as follows-in Section 2 we reformulate the classification given by [15] of the self-adjoint extensions of $-d^{2} / d x^{2}$ on $L^{2}[-a, a]$. In Section 3 , we classify these extensions in terms of some other sets of parameters, not considered in [15]. In Section 4, we construct the first SUSY partners for those extensions with positive ground level energy and give the precise form of its eigenfunctions. In Section 5, we give the complete sequence of SUSY partners for each of these extensions. We close this article with a Conclusions Section and an Appendix in which we show what the correct form for the wave functions for the energy levels should be.

\section{Self-Adjoint Extensions: Determination of Their Eigenvalues}

Let us go back to the differential operator $H_{0}:=-d^{2} / d x^{2}$ defined on $L^{2}[-a, a], a>0$ and with domain $\mathcal{D}_{0}$ as above, just before (1). On $\mathcal{D}_{0}, H_{0}$ is symmetric (Hermitian) with deficiency indices $(2,2)$ [15]. According to the von-Neumann theorem [25], $H_{0}$ admits an infinite number of self-adjoint extensions labeled by four real parameters.

The adjoint operator $H_{0}^{\dagger}$ acts as $-d^{2} / d x^{2}$ on the functions of its domain (see $[36,37]$ for a definition of the domain of the adjoint of a given densely defined operator and its properties). If $\phi$ is a function of such domain, we get integrating by parts:

$$
\left\langle-\frac{d^{2}}{d x^{2}} \phi, \phi\right\rangle=B(\phi, \phi)+\left\langle\phi,-\frac{d^{2}}{d x^{2}} \phi\right\rangle,
$$

where $\langle-,-\rangle$ denotes the scalar product on $L^{2}[-a, a]$ and

$$
B(\phi, \phi)=\phi^{\prime}(a) \phi^{*}(a)-\phi(a) \phi^{\prime *}(a)-\phi^{\prime}(-a) \phi^{*}(-a)+\phi(-a) \phi^{\prime *}(-a),
$$


the prime being the derivative with respect to the variable $x$ and the asterisk meaning complex conjugate. The self-adjoint extensions of $H_{0}$ are equal to $-d^{2} / d x^{2}$ as an operator acting on the subdomains of the domain of $H_{0}^{\dagger}$ of functions with $B(\phi, \phi)=0$. This happens if and only if there exists a $2 \times 2$ unitary matrix $U$ such that (see [15] and references quoted therein):

$$
\left(\begin{array}{c}
2 a \phi^{\prime}(-a)-i \phi(-a) \\
2 a \phi^{\prime}(a)+i \phi(a)
\end{array}\right)=U\left(\begin{array}{c}
2 a \phi^{\prime}(-a)+i \phi(-a) \\
2 a \phi^{\prime}(a)-i \phi(a)
\end{array}\right) .
$$

The set of self-adjoint extensions of $H_{0}$ is in one to one correspondence with the set of $2 \times 2$ unitary operators $U$. Thus, each of these extensions will be labeled by its corresponding operator as $H_{\alpha}$. Since there is a set of four real independent parameters that characterize the set of operators $U$, then, the set of self-adjoint extensions of $H_{\alpha}$ is also characterized by the same parameters [15]. Each of the operators $U$ has the following form [15]:

$$
U=e^{i \psi}\left(\begin{array}{cc}
m_{0}-i m_{3} & -m_{2}-i m_{1} \\
m_{2}-i m_{1} & m_{0}+i m_{3}
\end{array}\right)
$$

Here, $\psi$ and $m_{i}, i=0,1,2,3$ are real parameters so that $\psi \in[0, \pi]$ and $m_{0}^{2}+m_{1}^{2}+m_{2}^{2}+$ $m_{3}^{2}=1$, which means that only four parameters are independent [15]. The latter relation is a consequence of unitarity: the modulus of the determinant of $U$ must be a number of modulus one.

There are some of these extensions with a clear physical interest, which does not mean that the others are irrelevant from the physics point of view. In [15], the authors distinguish three categories of extensions:

(i) Those which preserve time reversal;

(ii) Those which preserve parity;

(iii) Those preserving positivity.

Apart from these three categories, there are some other extensions. The reason why the authors of [15] single out those extensions that preserve positivity is due to the existence of extensions with negative energies. In fact, as proven in [34] (Theorem 16, vol 2, page 44), $H_{\alpha}$ may have one (which may be doubly degenerate) or two (with no degeneration) negative energy states. All other extensions have non-negative eigenvalues and are called positivity preserving. Only three of these positivity preserving extensions with special simplicity are discussed in [15]. We want to determine the energy levels in this situation.

In order to obtain the energy levels for a specific self-adjoint extension, $H_{\alpha}$, of $H_{0}=-d^{2} / d x^{2}$ on $L^{2}[-a, a]$, we have to solve the Schrödinger equation and impose on its solutions the boundary conditions that characterize the extension. These boundary conditions are given by the (4) and (6). However as stated in [15], the determination of which operators $U$ satisfy the positivity condition as stated before involves tedious considerations. To circumvent this difficulty, let us consider the general solution of the time independent Scrödinger equation $-d^{2} \phi(x) / d x^{2}=E \phi(x)$, with $E=s^{2} /(2 a)^{2} \geq 0$, where $2 a$ is the infinite square well width (Although the energy is given, in our notation, by $\hbar^{2} E / 2 m$, we are calling "energy" the quantity represented by E.). This general solution is

$$
\phi(x)=A \cos \left(\frac{s x}{2 a}\right)+B \sin \left(\frac{s x}{2 a}\right) .
$$

Here, $A$ and $B$ have to be fixed with two conditions: (i) $\phi(x)$ should be normalized in $L^{2}[-a, a]$ and (ii) $\phi(x)$ should fulfill the boundary conditions (4) and (5) so that $E \geq 0$. 
Let us use (6) in relation (4) giving the general matching conditions, so as to obtain the following homogeneous linear system:

$$
(\mathcal{L}(s)-U \mathcal{M}(s))\left(\begin{array}{l}
A \\
B
\end{array}\right)=\mathcal{N}(s)\left(\begin{array}{l}
A \\
B
\end{array}\right)=0,
$$

where

$$
\mathcal{L}(s)=\left(\begin{array}{cc}
s \sin \frac{s}{2}-i \cos \frac{s}{2} & s \cos \frac{s}{2}+i \sin \frac{s}{2} \\
-s \sin \frac{s}{2}+i \cos \frac{s}{2} & s \cos \frac{s}{2}+i \sin \frac{s}{2}
\end{array}\right), \quad \mathcal{M}(s)=\left(\begin{array}{cc}
s \sin \frac{s}{2}+i \cos \frac{s}{2} & s \cos \frac{s}{2}-i \sin \frac{s}{2} \\
-s \sin \left(\frac{s}{2}-i \cos \frac{s}{2}\right. & s \cos \frac{s}{2} i \sin \frac{s}{2}
\end{array}\right) .
$$

The eigenvalues $\lambda_{ \pm}(s)$ of the matrix $\mathcal{N}(s)$ are given by

$$
\lambda_{ \pm}(s)=\frac{\operatorname{Tr}(\mathcal{N}(s))}{2} \pm \sqrt{\left(\frac{\operatorname{Tr}(\mathcal{N}(s))}{2}\right)^{2}-\operatorname{det}(\mathcal{N}(s))}
$$

The trace and the determinant of $\mathcal{N}(s)$ can be easily calculated and are, respectively:

$$
\operatorname{Tr}(\mathcal{N}(s))=e^{-\frac{1}{2} i(s-2 \psi)}\left(-m_{3}(s+1)+i m_{2} e^{i s}(s-1)\right),
$$

$$
\operatorname{det}(\mathcal{N}(s))=-4 i e^{i \psi}\left[\left(m_{0}+\cos \psi\right) \sin s+2 s\left(m_{1}-\cos s \sin \psi\right)-s^{2}\left(m_{0}-\cos \psi\right) \sin s\right] .
$$

To begin with, let us remark that in order to have non-trivial solutions of (7) we must have

$$
\operatorname{det}(\mathcal{N}(s))=0 .
$$

Then, the set of eigenvalues of $\mathcal{N}(s)$ is given by $\operatorname{Tr}(\mathcal{N}(s))$ and 0 , as may be immediately seen from (9). The condition (12) gives a relation between the values of the energy, determined by the real parameter $s$, since $E=s^{2} /(2 a)^{2}$, and the parameters $\psi, m_{0}$ and $m_{1}$, as in (5). In consequence, the energy levels depend on the values of these three parameters only. From (11) and (12), we obtain the following two transcendental equations (one with plus sign and the other with minus sign):

$$
s \sin s=\frac{m_{1}-\cos s \sin \psi}{m_{0}-\cos \psi} \pm \sqrt{\left(\frac{m_{1}-\cos s \sin \psi}{m_{0}-\cos \psi}\right)^{2}+\frac{m_{0}+\cos \psi}{m_{0}-\cos \psi} \sin ^{2} s} .
$$

This form of the transcendental equations is quite interesting, since it will serve as an efficient estimation of the energy levels when these values cannot be exactly calculated. Otherwise, they permit to obtain exact solutions whenever they exists. Let us now summarize three of the results provided by [15], which we will need later on:

- The eigenvector $(A, B)$ of $\mathcal{N}(s)$ with 0 eigenvalue is given by

$$
\begin{aligned}
& A=\left[i+e^{i \psi}\left(i m_{0}+m_{1}-i m_{2}+m_{3}\right)\right] \sin \frac{s}{2}+s\left[1+e^{i \psi}\left(m_{0}+i m_{1}+m_{2}+i m_{3}\right)\right] \cos \frac{s}{2} \\
& B=s\left[-1+e^{i \psi}\left(m_{0}+i m_{1}+m_{2}-i m_{3}\right)\right] \sin \frac{s}{2}+\left[i+e^{i \psi}\left(i m_{0}-m_{1}+i m_{2}+m_{3}\right)\right] \cos \frac{s}{2} .
\end{aligned}
$$

These expressions generalize similar ones published in citation [14] of our Reference [15]. We see that the eigenvector depends on all the parameters $\left(m_{0}, m_{1}, m_{2}, m_{3}, \psi\right)$.

- The extensions preserving time reversal invariance, are given by

$$
m_{2}=0 \text {. }
$$

- The parity preserving extensions of $H_{0}$ are those for which the eigenfunctions $\phi(x)$ verify :

$$
|\phi(x)|^{2}=|\phi(-x)|^{2} \Longrightarrow|\phi(a)|^{2}=|\phi(-a)|^{2} .
$$




\section{Parity Preserving Extensions of $\mathrm{H}_{0}$}

We are interested now in getting more information on the parity preserving extensions of $H_{0}$. Then, if we use (6) in (17) we obtain that $\operatorname{Re}\left(A B^{*}\right) \sin s=0$. Hence, either

$$
\sin s=0 \text { or } \operatorname{Re}\left(A B^{*}\right)=0 .
$$

Compare to Equation (68) in citation [14] of our Reference [15]. Taking into account the values of $(A, B)$ given in (14) and (15) and also the fact that $\operatorname{det}(\mathcal{N}(s))=0$, the second equation of (18) implies that either $m_{3}=0$ or

$$
\left(m_{3}+\sin \psi\right) \sin s+2 s\left(m_{2}+\cos s \cos \psi\right)+s^{2}\left(\sin \psi-m_{3}\right) \sin s=0 .
$$

Solving this equation as if it were a quadratic equation on $s$ gives a pair of transcendental equations which closely resemble Equation (13). Thus, the complete set of solutions of (18) are

$$
\begin{gathered}
m_{3}=0, \\
\sin s=0, \\
s \sin s=\frac{m_{2}+\cos s \cos \psi}{m_{3}-\sin \psi} \pm \sqrt{\left(\frac{m_{2}+\cos s \cos \psi}{m_{3}-\sin \psi}\right)^{2}+\frac{m_{3}+\sin \psi}{m_{3}-\sin \psi} \sin ^{2} s}
\end{gathered}
$$

Hence, when the parity is preserved, Equation (13) holds. This happens for three different situations given by Equations (20a)-(20c). These formulas, plus (13), which derives from such a general principle as $\operatorname{det}(\mathcal{N}(s))=0$, should give the energy levels for the infinite square well with parity preserving self-adjoint extensions, $H_{\alpha}$, of $H_{0}$.

Equation (20a) does not provide any extra information, (13) being the only relation which gives information on the energy spectrum. This parity preserving condition $m_{3}=0$ has been already used in [15], although in this paper relations (20b) and (20c) are not mentioned.

Equation (20b) obviously gives an energy spectrum of the parity preserving extensions that coincides to the spectrum given by texts in Quantum Mechanics for the extension with domain given by functions with $\phi(-a)=\phi(a)=0$. Henceforth, we shall call this extension the textbook extension.

Finally, (20c) gives the energy levels for other parity preserving extensions in terms of the three parameters $\left(\psi, m_{2}, m_{3}\right)$.

In consequence, we have eight different situations for those extension having a nonnegative spectrum, including those with time reversal and parity invariance, as shown in Table 1. In the next section we will analyze some of these situations.

Table 1. List of how to obtain the possible spectra as a function of the conserved properties.

\begin{tabular}{ccc}
\hline & Generic Spectrum: (13) & \\
\hline & Time reversal invariance: (13) and (16), or $m_{2}=0$ \\
\cline { 2 - 3 } & $(13)$ and (20a) & $m_{2}=0$ \\
\cline { 2 - 3 } Parity preserving: & $(13)$ and (20b) & $m_{2} \neq 0$ \\
\cline { 2 - 3 } & $(13)$ and (20c) & $m_{2}=0$ \\
\cline { 2 - 3 } & & $m_{2} \neq 0$ \\
\hline
\end{tabular}




\section{Spectrum of the Free Particle on a Finite Interval}

One of the goals of our study is to solve the eigenvalue problem for all the self-adjoint extensions, $H_{\alpha}$, of the operator $H_{0}=-d^{2} / d x^{2}$ on $L^{2}[-a, a]$, which from the point of view of the physicist is the infinite square well with width $2 a$. As we have already seen, there are only a few of these extensions for which we may obtain an exact solution, including the textbook extension. For most of these extensions the energy levels are solutions of a transcendental equation and, therefore, no explicit solutions of the eigenvalue problem for these extensions can be given.

\subsection{The Angular Representation of the Self-Adjoint Extensions of $H_{0}$}

Due to the relation between the parameters $m_{i}$, given by

$$
m_{0}^{2}+m_{1}^{2}+m_{2}^{2}+m_{3}^{2}=1,
$$

a new parametric representation of the self-adjoint extensions, $H_{\alpha}$, of $H_{0}=-d^{2} / d x^{2}$ on $L^{2}[-a, a]$ in terms of angular variables only is possible. Apart from the variable $\psi$, which is already angular, so that we keep it untouched, we have three other angular variables, $\theta_{i}$, $i=0,1,2$, defined by means of the following relations:

$$
m_{0}=\cos \theta_{1} \cos \theta_{0}, \quad m_{1}=\cos \theta_{1} \sin \theta_{0}, \quad m_{2}=\sin \theta_{1} \cos \theta_{2}, \quad m_{3}=\sin \theta_{1} \sin \theta_{2} .
$$

Taking into account that Equation (13) gives the values of $s$, and hence the energy levels, in terms of the triplet of parameters $\left(\psi, m_{0}, m_{1}\right)$, then, according to (22), $s$ will depend on the angular variables $\left(\psi, \theta_{0}, \theta_{1}\right)$ only. In general, we cannot solve (13) to find $s\left(\psi, \theta_{0}, \theta_{1}\right)$ explicitly. Since (13) depends on four parameters $\left(s, \psi, \theta_{0}, \theta_{1}\right)$, we cannot represent this equation in general but, as in Figure 1, we can plot the square root of the energy (essentially s) for given values of $\theta_{1}$ and $\sin \theta_{0}$ as a function of $\psi$.
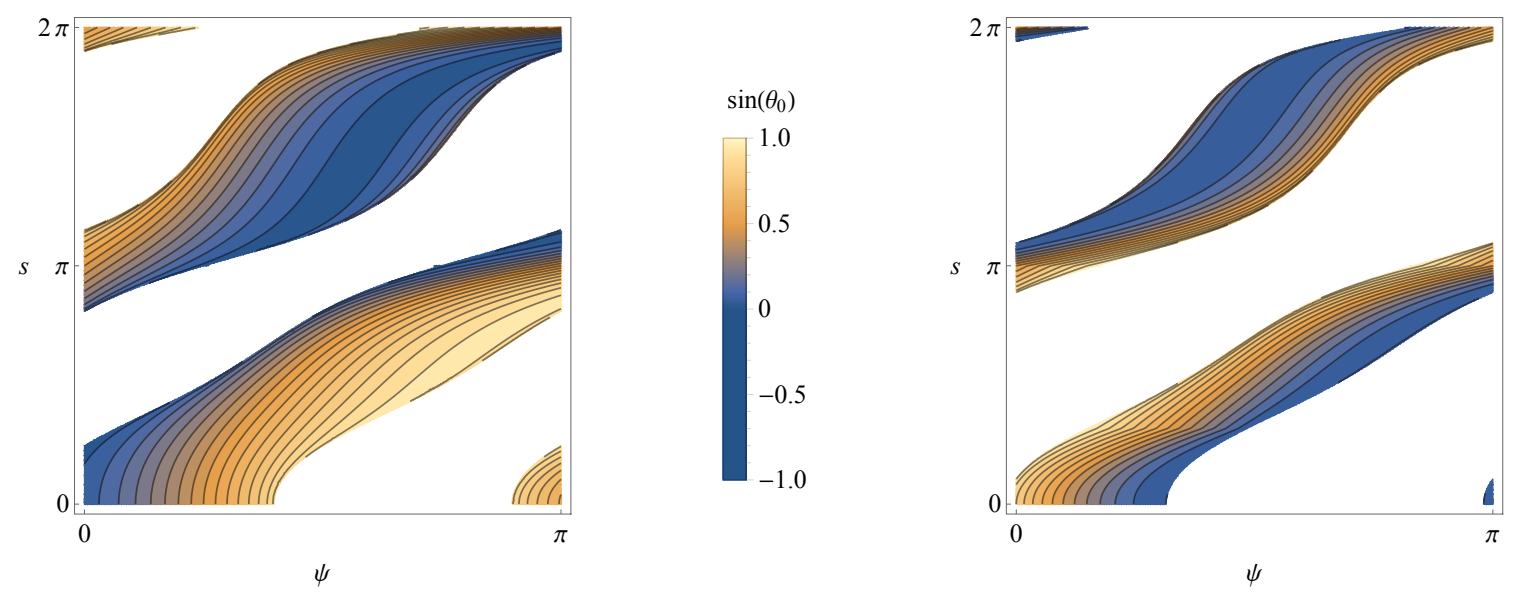

Figure 1. Two plots of the implicit Equation (13) with the parametrization (22) allow us to see the variation of the parameter $s$ (remember that $E=s^{2} /(2 a)^{2}$ ) as a function of $\psi$ and $\sin \theta_{0}$ : on the left for $\theta_{1}=\pi / 4$, on the right for $\theta_{1}=4 \pi / 3$.

The general case can neither be explicitly solved nor represented graphically. Yet, there are two other situations sharing this negative characteristics. One is $m_{2}=0$ (time invariance only) and $m_{3}=0$ (parity conservation only). All other cases either can be explicitly or graphically solved or both.

\subsection{Some Simple Cases}

In the sequel we are going to deal with the cases of Table 1 that can be treated in some way, either graphically or analytically. 


\subsubsection{Parity and Time Reversal Invariance: $m_{2}=m_{3}=0$}

For $m_{2}=m_{3}=0$, we have in (21) that $m_{0}^{2}+m_{1}^{2}=1$, that is, we can take $\theta_{1}=0$ in (22) and therefore $m_{0}=\cos \theta_{0}$ and $m_{1}=\sin \theta_{0}$. Then, let us go back to (10), so as to see that $\operatorname{Tr}(\mathcal{N}(s))=0$. Since one of the eigenvalues of $\mathcal{N}(s)$ must be zero, the fact that $\operatorname{Tr}(\mathcal{N}(s))=0$ makes the second eigenvalue also equal to zero. Thus, the matrix $\mathcal{N}(s)$ admits a Jordan decomposition in terms of an upper triangular matrix. Now, the transcendental Equation (13) becomes much simpler, still depending on the sign, \pm , of the square root. Note that this sign is positive if $s \in\{(0, \pi),(2 \pi, 3 \pi) .$.$\} and negative if s \in\{(\pi, 2 \pi),(3 \pi, 4 \pi), .$.$\} . In these$ two situations, the spectral equation, the vector $(A, B)$ and the eigenfunctions have the following explicit forms:

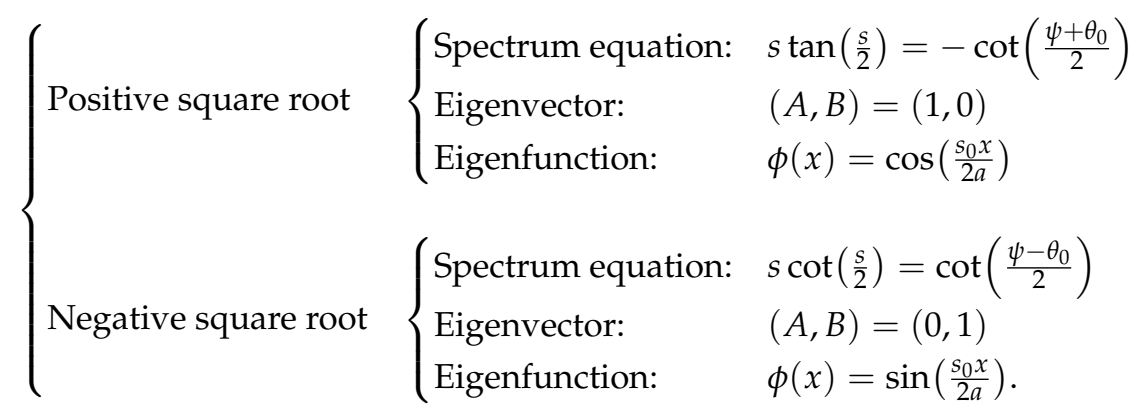

In the above expressions for the spectral equations, we may write $\frac{\psi-\theta_{0}}{2}=\varphi_{1}$ and $\frac{\psi+\theta_{0}}{2}=\varphi_{2}$, where $\varphi_{i}, i=1,2$ are two independent angles. Both spectral equations are represented in Figure 2. The combination of both solutions tend to the textbook solution in the limit $\varphi_{1,2} \rightarrow 0$ for both angles. The ground state for the textbook solution comes from the lowest state for the even parity preserving extensions. It is remarkable that if $\varphi_{1} \geq \arctan (1 / 2):=$ $\gamma$, then the ground state no longer comes from the even but from the odd parity preserving extensions, as can be clearly seen in Figure 2.

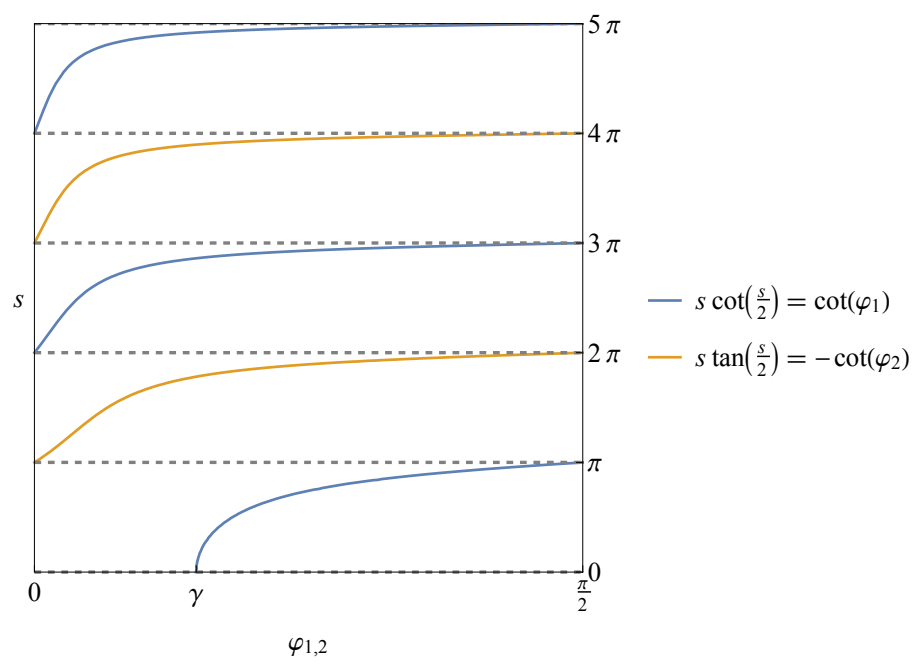

Figure 2. Energy levels $\left(E=s^{2} /(2 a)^{2}\right)$ for odd parity (blue) and even parity (yellow) solution for $m_{2}=m_{3}=0$, coming from (23).

\subsubsection{Parity Preserving Extensions Fulfilling $\sin s=0$}

In this situation, Equation (20b) implies that $s=n \pi$ with $n=0, \pm 1, \pm 2, \ldots$, so that $E=\left(n^{2} \pi^{2}\right) /(2 a)^{2}, n=1,2, \ldots$ The energy levels of all these extensions are the same as in the textbook's extension. No negative energy states may exist. In order to obtain the corresponding eigenfunctions, which may be different from those obtained for the textbook case, we parametrize these extensions using angular variables. However, we are now using 
a different angle parameterization from (22). Indeed, taking into account (13), we will find it very useful to use this one:

$$
\begin{aligned}
m_{0}=\cos \psi \cos \beta_{0}, & m_{1}=(-1)^{n} \sin \psi, \\
m_{2}=\cos \psi \sin \beta_{0} \cos \beta_{1}, & m_{3}=\cos \psi \sin \beta_{0} \sin \beta_{1},
\end{aligned}
$$

where the exponent $n$ appearing in the expression for $m_{1}$ is the same number that labels $s$.

The eigenfunctions must obey the Schrödinger equation, so that they should be of the form (6). The coefficients $A$ and $B$ depend on the energy levels and, therefore, should be functions of $s$. In the simple case in which $s$ be a even or odd multiple of $2 \pi$, these coefficients can be obtained from the following relations, using (14) and (15) and the new parameterization:

$$
\begin{cases}s=2 q \pi: & \left\{\begin{array}{l}
A(s)=2 \pi q\left(1+e^{i \beta_{1}} \sin \beta_{0}-\cos \beta_{0}\right), \\
B(s)=i\left(1+e^{-i \beta_{1}} \sin \beta_{0}+\cos \beta_{0}\right),
\end{array} \quad q=1,2, \ldots\right. \\
s=(2 q+1) \pi: & \left\{\begin{array}{l}
A(s)=i\left(1-e^{i \beta_{1}} \sin \beta_{0}+\cos \beta_{0}\right), \\
B(s)=\pi(2 q+1)\left(-1+e^{-i \beta_{1}} \sin \beta_{0}+\cos \beta_{0}\right),
\end{array} \quad q=0,1, \ldots\right.\end{cases}
$$

The eigenfunctions depend on the angles $\left(\psi, \beta_{0}, \beta_{1}\right)$ only. These angles are those defined in (24) and (25); note the difference with the angles defined in (22). If we take the limits $\beta_{0} \rightarrow 0$ and $\beta_{1} \rightarrow 0$, we recover the eigenfunctions for the textbook extension.

\subsubsection{Parity and Time Reversal Invariance Extensions Fulfilling (20c)}

We have seen that (13) has a general validity, which is independent of the particular situation under study. On the other hand, (20c) is valid for extensions that preserve parity invariance. Note that the left hand side in both equations is the same: $s \sin (s)$. This suggests that the identity between both right hand sides would help to solve the spectral equation in this case. This identity gives:

$$
\begin{aligned}
& \frac{m_{1}-\cos s \sin \psi}{m_{0}-\cos \psi} \pm \sqrt{\left(\frac{m_{1}-\cos s \sin \psi}{m_{0}-\cos \psi}\right)^{2}+\frac{m_{0}+\cos \psi}{m_{0}-\cos \psi} \sin ^{2} s} \\
& \quad=\frac{m_{2}+\cos s \cos \psi}{m_{3}-\sin \psi} \pm \sqrt{\left(\frac{m_{2}+\cos s \cos \psi}{m_{3}-\sin \psi}\right)^{2}+\frac{m_{3}+\sin \psi}{m_{3}-\sin \psi} \sin ^{2} s}
\end{aligned}
$$

which may be written in polynomial form as $\cos ^{4} s+P_{1} \cos ^{3} s+P_{2} \cos ^{2} s+P_{3} \cos s+P_{4}=0$, where the functions $P_{i}$ depend on the parameters $\left(m_{0}, m_{1}, m_{2}, m_{3}, \psi\right)$. We do not write the precise form of this polynomial relation in here, since it is extremely long and it does not show interesting features. Nevertheless, it is important to note that this is a fourth order polynomial on the variable $\cos s$ with coefficients depending on the parameters. Two of these solutions of (27) are

$$
\cos s= \pm 1
$$

These solutions may be written as $\sin s=0$, which coincide with (20b), so that no new solutions for the spectral problem arise from (28). The other two solutions are quadratic as function of the parameters and are rather huge and intractable. To simplify this problem as much as possible, let us define a third and last angle re-parameterization of the $m_{i}$ :

$m_{0}=\sin \omega_{1} \cos \omega_{2}, \quad m_{1}=\cos \omega_{1} \sin \omega_{0}, \quad m_{2}=\cos \omega_{1} \cos \omega_{0}, \quad m_{3}=\sin \omega_{1} \sin \omega_{2}$.

This parameterization is quite similar to (22), where we have interchanged the expressions for $m_{0}$ and $m_{2}$. In terms of the new angular variables, an expression for the energy levels as functions of $s$ is given by 


$$
\begin{aligned}
\cos s=- & \cos \omega_{1} \cos \left(\omega_{0}+\omega_{2}\right) \cos \left(\omega_{2}-\psi\right) \\
& -\sec \omega_{1}\left[\sin \left(\omega_{0}+\omega_{2}\right) \sin \left(\omega_{2}-\psi\right) \pm i \sqrt{\sin ^{4} \omega_{1} \cos ^{2}\left(\omega_{0}+\omega_{2}\right) \sin ^{2}\left(\omega_{2}-\psi\right)}\right]
\end{aligned}
$$

As we want $s$ to be real (in order to have positive eigenvalues of the energy), the imaginary term in (30) must vanish. Note that all factors under the square root are positive, so that the eigenvalues of the energy can be found, with all those in Equation (30) for each of the factors under the square root vanishing. There are three possibilities, which yield the following equations:

$$
\begin{gathered}
\sin \omega_{1}=0 \Longrightarrow \cos s= \pm \cos \left(\omega_{0}+\psi\right) \Longrightarrow s=n \pi \pm\left(\omega_{0}+\psi\right) \\
\cos \left(\omega_{0}+\omega_{2}\right)=0 \Longrightarrow \cos s= \pm \sec \omega_{1} \sin \left(\omega_{2}-\psi\right) \\
\sin \left(\omega_{2}-\psi\right)=0 \Longrightarrow \cos s= \pm \cos \omega_{1} \cos \left(\omega_{0}+\psi\right)
\end{gathered}
$$

Equations (31a) and (31c), give rise to an equally spaced spectrum on the variable $s$ (not for the energy), for which $s=n \pi+f\left(\omega_{0}, \omega_{1}, \psi\right), n=0,1,2, \ldots$ In any case, the minimal energy level is given by $f\left(\omega_{0}, \omega_{1}, \psi\right)$. The determination of this minimal energy is not a trivial matter for (31c), since its solution $s=\arccos \left(\cos \left(\omega_{1}\right) \cos \left(\omega_{0}+\psi\right)\right)$ is given by a multi-valued function.

Equation (31b) is even more problematic, as its right hand side may be bigger than one in modulus. One may think that this formula provides the negative energy values for $|\cos s|>1$. However, we have to keep in mind that there are only possible two negative energy levels, if any, or if there is only one, this could be either single or doubly-degenerate, so that (31b) may not give solutions to the energy spectrum and should be discarded, in principle.

\subsection{About the Negative and Zero Energies}

Up to now, we have not been interested in zero and negative values of the energy. Observe that the transcendental equation (13), which gives the energy levels, is valid for those extensions, $H_{\alpha}$, having positive energies only. These energy levels are, in all positive energy cases, infinite.

If we wanted to analyze those Hamiltonians $H_{\alpha}$ with negative energy levels, we need to perform the replacement $s \rightarrow-i r$ in the wave function (6) as well as in (13). The latter appears in terms of hyperbolic functions and may have one or two solutions with zero or negative energies. If there were just one negative energy level, this is doubly degenerate [34].

When the ground state shows a negative energy, its wave function is similar to (6), where the trigonometric functions have been replaced by hyperbolic functions. In this case, the ground state wave functions may have zeros (nodes) on the interval $[-a, a]$. Here, the general formalism says that the procedure to obtain the SUSY partners is not valid [1]. Nevertheless, this formalism gives a procedure and this procedure may still be applied in this case. The result is clear-instead of obtaining a new potential with a countable infinite number of equally spaced values of the square root of the eigenvalues of the Hamiltonian (s), we obtain new Hamiltonians with either a finite number of eigenvalues or a continuous spectrum only. In the first case, these energy eigenvalues come from a transcendental equation. In the second case, partner potentials are often singular, showing an infinite divergence. We shall discuss this situation in detail in a forthcoming publication. A similar situation emerges when the ground state has zero energy.

From now on, we will concentrate in obtaining the SUSY partners of the self-adjoint extensions that we have analyzed up to now. 


\section{Supersymmetric Partners for the Simplest Extensions}

In this section we shall consider the first and second order supersymmetry transformation applied to some of the self-adjoint extensions $H_{\alpha}$ so far considered in here.

\subsection{First Order SUSY Partners}

The technique to obtain the SUSY partner corresponding to a given self-adjoint operator with discrete spectrum has been discussed in [1]. To begin with, let us fix some notation and call $H_{\alpha}$ to the self-adjoint extension characterized by the values $\alpha:=\left(m_{0}, m_{1}, m_{2}, m_{3}, \psi\right)$ of the parameters.

Then, let us follow the procedure of [1] to obtain the SUSY partners of $H_{\alpha}$. First of all, we need to determine the ground state $\phi_{\alpha}^{(0)}(x)$ of $H_{\alpha}$. This ground state has energy $E_{\alpha}^{(0)}=\left(s_{\alpha}^{(0)} /(2 a)\right)^{2}$, which may be in principle either positive or negative. In the present paper, we shall deal with those extensions having the ground level with positive energy and, for all energy levels, $s_{\alpha}^{(n)}, n=0,1,2, \ldots$, we have $s_{\alpha}^{(n)}=(n+1) s_{\alpha}^{(0)}$.

In general, there are two supersymmetric partners of the self-adjoint extension $H_{\alpha}$, which are Hamiltonians of the form $-d^{2} / d x^{2}+V_{\alpha}^{(j)}$, where $V_{\alpha}^{(j)}, j=1,2$, are a pair of new potentials which is called partner potentials. In order to obtain each of the $V_{\alpha}^{(j)}$, pick the ground state $\phi_{\alpha}^{(0)}(x)$ of $H_{\alpha}$. The explicit form of this ground state is, after (6),

$$
\phi_{\alpha}^{(0)}(x)=A\left(s_{0}\right) \cos \left(\frac{s_{0}}{2 a} x\right)+B\left(s_{0}\right) \sin \left(\frac{s_{0}}{2 a} x\right),
$$

where we have used the simplified notation $s_{0}:=s_{\alpha}^{(0)}$, which we shall henceforth keep for simplicity unless otherwise stated. Since (32) must be in the domain of $H_{\alpha}$, the coefficients $A\left(s_{0}\right)$ and $B\left(s_{0}\right)$ must satisfy the boundary conditions defining this domain. Although these coefficients depend on the energy ground state $s_{0}$, we shall also omit this dependence, unless necessary. Then, we construct the partner potentials $V_{\alpha}^{(j)}, j=1,2$ using an intermediate function called the super-potential, $W_{\alpha}(x)$, which is defined as

$$
W_{\alpha}(x):=-\frac{\partial_{x} \phi_{\alpha}^{(0)}(x)}{\phi_{\alpha}^{(0)}(x)},
$$

where $\partial_{x}$ means derivative with respect to $x$.

Now, we construct the partner potentials $V_{\alpha}^{(j)}, j=1,2$, as [1]

$$
\begin{aligned}
& V_{\alpha}^{(1)}(x)=W_{\alpha}^{2}(x)-W_{\alpha}^{\prime}(x)=-\left(\frac{s_{0}}{2 a}\right)^{2} \\
& V_{\alpha}^{(2)}(x)=W_{\alpha}^{2}(x)+W_{\alpha}^{\prime}(x)=\left(\frac{s_{0}}{2 a}\right)^{2}\left(1+2\left(\frac{A \sin \left(\frac{s_{0}}{2 a} x\right)-B \cos \left(\frac{s_{0}}{2 a} x\right)}{A \cos \left(\frac{s_{0}}{2 a} x\right)+B \sin \left(\frac{s_{0}}{2 a} x\right)}\right)^{2}\right) .
\end{aligned}
$$

According to (34), it comes that $V_{\alpha}^{(1)}(x)$ is constant and equal, in modulus, to the original system lowest energy level. We see that this solution is trivial, as only shifts the energy levels. If we represent as $\phi^{(1)}(x)$ and $E_{n}^{(1)}$ the wave function of the ground state and the $n$-th energy level in this situation, we have that

$$
\phi^{(1)}(x) \equiv \phi^{(0)}(x), \quad E_{n}^{(1)}=\left(\frac{s_{0}}{2 a}\right)^{2}\left(n^{2}-1\right) \equiv E_{n}^{(0)}-E_{n=1}^{(0)},
$$

and $n=1,2, \ldots$ is arbitrary. In the sequel, we omit the subindex $\alpha$ for simplicity in the notation, unless otherwise stated for necessity.

The Schrödinger equation coming from the second potential in (35) is 
$-\frac{d^{2}}{d x^{2}} \phi^{(2)}(x)+\left(\frac{s_{0}}{2 a}\right)^{2}\left(1+2\left(\frac{A \sin \left(\frac{s_{0}}{2 a} x\right)-B \cos \left(\frac{s_{0}}{2 a} x\right)}{A \cos \left(\frac{s_{0}}{2 a} x\right)+B \sin \left(\frac{s_{0}}{2 a} x\right)}\right)^{2}\right) \phi^{(2)}(x)=E_{n}^{(1)} \phi^{(2)}(x)$,

where the meaning of $\phi^{(2)}(x)$ is obvious. Next, let us define a new variable $z$ as

$$
\frac{s_{0}}{2 a} z=i W(x)=\frac{i s_{0}}{2 a} \frac{A \sin \left(\frac{s_{0}}{2 a} x\right)-B \cos \left(\frac{s_{0}}{2 a} x\right)}{A \cos \left(\frac{s_{0}}{2 a} x\right)+B \sin \left(\frac{s_{0}}{2 a} x\right)} .
$$

Under this change of variables, the Schrödinger Equation (37) takes the form:

$$
\left(1-z^{2}\right) \partial_{z}^{2} \phi^{(2)}(z)-2 z \partial_{z} \phi^{(2)}(z)+\left(\ell(\ell+1)-\frac{n^{2}}{1-z^{2}}\right) \phi^{(2)}(z)=0, \quad \text { with } \quad \ell=1,
$$

where $\partial_{z}$ represents the derivation with respect to $z$. This is a particular case of associated Legendre equation when $\ell=1$, and their solutions are well known. One of them is given by the associated Legendre functions of second kind:

$$
Q_{\ell}^{n}(z):=(-1)^{n}\left(1-z^{2}\right)^{n / 2} \frac{d^{n}}{d z^{n}} Q_{\ell}(z),
$$

where $Q_{\ell}(z)$ are the Legendre functions of the second kind [38]. These solutions for (39) provide the solutions for (37):

$$
\phi_{n}^{(2)}(x)=Q_{1}^{n}\left(i \frac{A \sin \left(\frac{s_{0}}{2 a} x\right)-B \cos \left(\frac{s_{0}}{2 a} x\right)}{A \cos \left(\frac{s_{0}}{2 a} x\right)+B \sin \left(\frac{s_{0}}{2 a} x\right)}\right),
$$

where, of course, we have taken the value $\ell=1$.

In addition, there is another set of solutions given by the first kind associated Legendre functions $P_{1}^{n}(z)$. These functions have not been considered as solutions to our problem, since they show singularities within the open interval $(-a, a)$ and are not square integrable, as shown in Appendix A. In Figure 3, we represent some of the wave equations just obtained in (41) for the lowest energy levels. Let us consider now the second partner Hamiltonian, $H_{\alpha}^{(2)}:=H_{\alpha}+V_{\alpha}^{(2)}$, or $H^{(2)}$ in brief. The solution $Q_{1}^{1}(z)$ shows a logarithmic singularity at each of its extremes and, therefore, it is not square integrable. Nevertheless, for $n \geq 2$, these solutions are square integrable, as is proved in the Appendix A.

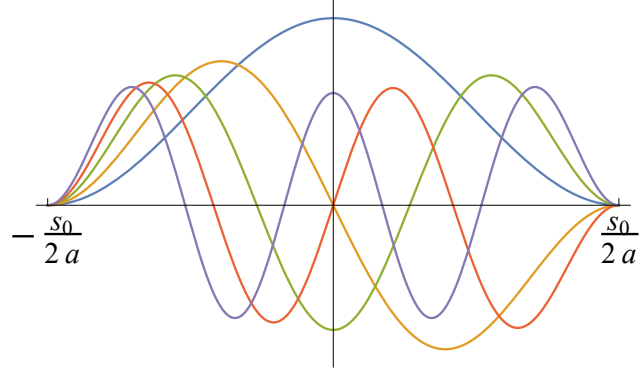

$$
\begin{aligned}
-n & =2 \\
-n & =3 \\
-n & =4 \\
-n & =5 \\
-n & =6
\end{aligned}
$$

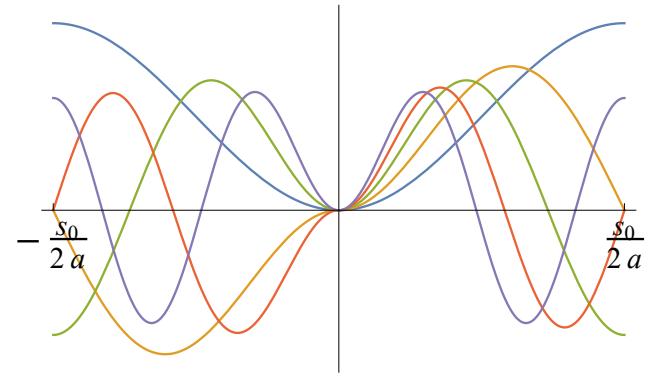

Figure 3. First order supersymmetric (SUSY) states $\phi_{n}^{(2)}(x)$ from (41) when the ground state of the original system is either purely even, that is $B=0$ (plot on the left), or purely odd, that is $A=0$ (plot on the right). Note that the quantum number $n$ of the Legendre function in (41) is the number of the nodes of the function.

\subsection{Second Order SUSY Partners}

Once we have obtained the first order SUSY partners for the self-adjoint extensions $H_{\alpha}$ with ground state of positive energy, let us inspect how we may obtain an infinite chain 
of higher order partners for $H_{\alpha}$. In the all above discussed cases, the bound state has wave function given by $Q_{1}^{2}(z)$ (which is obviously not the same for all cases, since the definition of $z$ changes). Then, we obtain the super-potential $W^{(2)}(x)$ by replacing $\phi_{n=1}^{(0)}(x)$ by $Q_{1}^{2}(z)$ (and then write $z$ in terms of $x$ ) in (33). This procedure gives rise to two second order potential partner candidates, which are:

$$
\begin{aligned}
& V^{(2,1)}(x)=W_{(2)}^{2}(x)-W_{(2)}^{\prime}(x)=\left(\frac{s_{0}}{2 a}\right)^{2}\left(1+2\left(\frac{A \sin \left(\frac{s_{0}}{2 a} x\right)-B \cos \left(\frac{s_{0}}{2 a} x\right)}{A \cos \left(\frac{s_{0}}{2 a} x\right)+B \sin \left(\frac{s_{0}}{2 a} x\right)}\right)^{2}\right)-3\left(\frac{s_{0}}{2 a}\right)^{2}, \\
& V^{(2,2)}(x)=W_{(2)}^{2}(x)+W_{(2)}^{\prime}(x)=\left(\frac{s_{0}}{2 a}\right)^{2}\left(1+3\left(\frac{A \sin \left(\frac{s_{0}}{2 a} x\right)-B \cos \left(\frac{s_{0}}{2 a} x\right)}{A \cos \left(\frac{s_{0}}{2 a} x\right)+B \sin \left(\frac{s_{0}}{2 a} x\right)}\right)^{2}\right) .
\end{aligned}
$$

Although the notation used in (42) and (43) should be clear, we need a generalization of it, as we are going to consider further order partners next. Thus, we shall use $V^{(i, j)}(x)$ and $W_{(i)}(x)$, where the index $i$ gives the order of the partner, which in the above case is $i=2$. This index may take all possible values $i=1,2,3, \ldots$ The index $j$ always takes two possible values, $j=1,2$. From this point of view, $V_{\alpha}^{(i)}(x)$ in (34) could be written as $V^{(1, i)}(x)$. Analogously, we may use for the $i$-th partner Hamiltonian the notation $H^{(i, j)}$. To simplify the notation, we have always omitted the subindex $\alpha$, which labels the precise self-adjoint extension we are considering.

Observe that according to (34) and (42), $V^{(2,1)}(x)=V^{(1,2)}(x)-3\left(s_{0} /(2 a)\right)^{2}=V_{\alpha}^{(2)}-$ $3\left(s_{0} /(2 a)\right)^{2}$, so that $H^{(2,1)}$ and $H^{(1,2)}$ have the same eigenvalues shifted by $3\left(s_{0} /(2 a)\right)^{2}$. Thus, we ignore (42) and solely consider (43). For (43), we may do a similar analysis than in the previous case, that is, first order SUSY partner, so that the bound state wave functions are given by

$$
\phi_{n}^{(2,2)}(x)=Q_{2}^{n}\left(i \frac{A \sin \left(\frac{s_{0}}{2 a} x\right)-B \cos \left(\frac{s_{0}}{2 a} x\right)}{A \cos \left(\frac{s_{0}}{2 a} x\right)+B \sin \left(\frac{s_{0}}{2 a} x\right)}\right) .
$$

In this second order SUSY, both functions $Q_{2}^{1}(z)$ and $Q_{2}^{2}(z)$ have logarithmic singularities at the points $x= \pm a$, so that they are not square integrable on $[-a, a]$ and, consequently, should be discarded as proper eigenfunctions of $H^{(2,2)}$. Thus, the ground state for $H^{(2,2)}$ has a wave function given by $Q_{2}^{3}(z)$. This is a general behaviour that could be checked at each step going from a SUSY partner to the next one, as is shown in Figure 4.
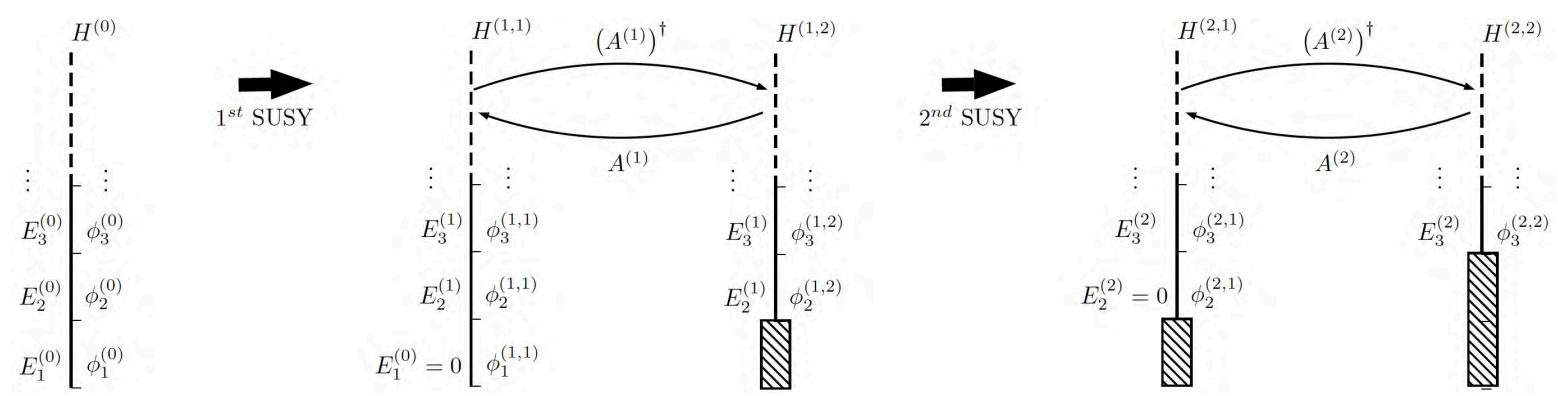

Figure 4. Different energy levels of first and second supersymmetry Hamiltonians.

\section{Supersymmetric Self-Adjoint Extensions of the Infinite Well at $\ell$-Order} ing:

Let us begin this Section with a summary of the notation employed so far and its mean- 


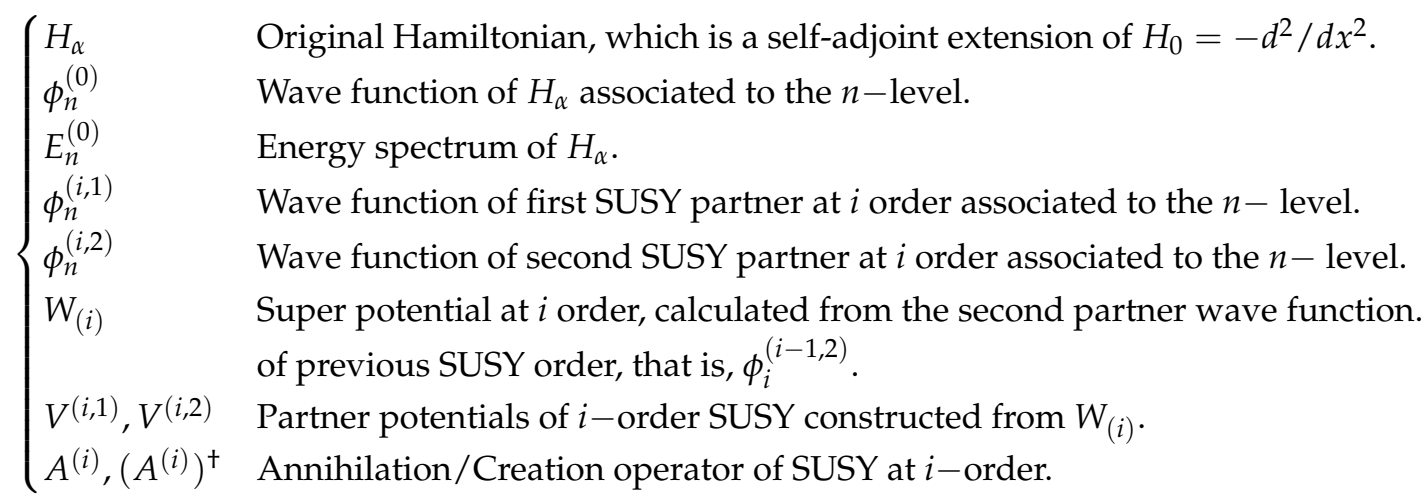

Creation $\left(A^{(i)}\right)^{\dagger}$ and annihilation $A^{(i)}$ operators will be defined later.

So far, we have obtained potentials and wave functions for the first and second SUSY partners for self-adjoint extensions of $H_{0}=-d^{2} / d x^{2}$ with ground level of positive energy. With the help of the induction method, we may find potentials as well as wave functions and energy levels for arbitrary order $\ell$ SUSY partners for the same class of self-adjoint extensions. We have seen already that from the SUSY partners $V^{(i, 1)}, V^{(i, 2)}$, only the last one is really interesting and we will focus on it in the sequel.

In order to apply the inductive method, let us assume that the ground state for the $\ell$-th SUSY partner, $H^{(\ell, 2)}$, of $H_{\alpha}$ is given by

$$
\phi_{\ell+1}^{(\ell, 2)}(x)=Q_{\ell}^{\ell+1}\left(i \frac{A \sin \left(\frac{s_{0}}{2 a} x\right)-B \cos \left(\frac{s_{0}}{2 a} x\right)}{A \cos \left(\frac{s_{0}}{2 a} x\right)+B \sin \left(\frac{s_{0}}{2 a} x\right)}\right),
$$

as in the previous cases (41) and (44). Then, the super-potential takes the following form:

$$
W_{(\ell+1)}=-\frac{\partial_{x}^{2} \phi^{(\ell, 2)}(x)}{\phi^{(\ell, 2)}(x)}=\frac{s_{0}(\ell+1)}{2 a}\left(\frac{A \sin \left(\frac{s_{0}}{2 a} x\right)-B \cos \left(\frac{s_{0}}{2 a} x\right)}{A \cos \left(\frac{s_{0}}{2 a} x\right)+B \sin \left(\frac{s_{0}}{2 a} x\right)}\right)=-i \frac{s_{0}(\ell+1)}{2 a} z,
$$

where $\partial_{x}^{2}$ denotes the second derivative with respect to the variable $x$. Once we have the super potential, we readily obtain the partner potentials at $\ell+1$ order, which are

$$
\begin{aligned}
& V^{(\ell+1,1)}(x)=\left(W_{(\ell+1)}\right)^{2}-\partial_{x} W_{(\ell+1)}=\frac{s_{0}^{2}(\ell+1)}{(2 a)^{2}}\left(-1+\ell\left(\frac{A \sin \left(\frac{s_{0}}{2 a} x\right)-B \cos \left(\frac{s_{0}}{2 a} x\right)}{A \cos \left(\frac{s_{0}}{2 a} x\right)+B \sin \left(\frac{s_{0}}{2 a} x\right)}\right)^{2}\right), \\
& V^{(\ell+1,2)}(x)=\left(W_{(\ell+1)}\right)^{2}+\partial_{x} W_{(\ell+1)}=\frac{s_{0}^{2}(\ell+1)}{(2 a)^{2}}\left(1+(\ell+2)\left(\frac{A \sin \left(\frac{s_{0}}{2 a} x\right)-B \cos \left(\frac{s_{0}}{2 a} x\right)}{A \cos \left(\frac{s_{0}}{2 a} x\right)+B \sin \left(\frac{s_{0}}{2 a} x\right)}\right)^{2}\right) .
\end{aligned}
$$

Note that although the label $\alpha$ is not written explicitly on the above equations and many others, potentials and wave functions must depend on $\alpha$. This dependence is hidden in $s_{0}$, where we have not made it explicitly for simplicity in the notation.

The Schrödinger equation for the first $(\ell+1)$-th order partner potential, $V^{(\ell+1,1)}$, is

$$
-\partial_{x}^{2} \phi^{(\ell+1,1)}(x)+V^{(\ell+1,1)}(x) \phi^{(\ell+1,1)}(x)=E^{(\ell+1)} \phi^{(\ell+1,1)}(x) .
$$

If we change it to the $z$ variable, (49) takes the form:

$$
\left(1-z^{2}\right)^{2} \partial_{z}^{2} \phi^{(\ell+1,1)}(z)-2 z\left(1-z^{2}\right) \partial_{z} \phi^{(\ell+1,1)}(z)-(\ell+1)\left(1+\ell z^{2}\right) \phi^{(\ell+1,1)}(x)=\left(\frac{2 a}{s_{0}}\right)^{2} E^{(\ell+1)} \phi^{(\ell+1,1)}(x) .
$$

Equation (50) is a new Legendre-type equation for which solutions are known. The respective eigenfunctions and eigenvalues are 


$$
\phi_{n}^{(\ell+1,1)}(x)=Q_{\ell}^{n}\left(i \frac{A \sin \left(\frac{s_{0}}{2 a} x\right)-B \cos \left(\frac{s_{0}}{2 a} x\right)}{A \cos \left(\frac{s_{0}}{2 a} x\right)+B \sin \left(\frac{s_{0}}{2 a} x\right)}\right), \quad E_{n}^{(\ell+1)}=\left(\frac{s_{0}}{2 a}\right)^{2}\left(n^{2}-(\ell+1)^{2}\right) .
$$

Observe that the first partner wave functions of order $\ell+1$ are the same as the second partner wave functions of order $\ell$, that is, $\phi_{n}^{(\ell+1,1)}(x) \equiv \phi_{n}^{(\ell, 2)}(x)$. The Schrödinger equation with potential $V^{(\ell+1,2)}(x)$ is

$$
-\partial_{x}^{2} \phi^{(\ell+1,2)}(x)+V^{(\ell+1,2)}(x) \phi^{(\ell+1,2)}(x)=E_{n}^{(\ell+1)} \phi^{(\ell+1,2)}(x),
$$

which in terms of the $z$ variable becomes:

$$
\left(1-z^{2}\right)^{2} \partial_{z}^{2} \phi^{(\ell+1,2)}(z)-2 z\left(1-z^{2}\right) \partial_{z} \phi^{(\ell+1,2)}(z)+(\ell+1)\left(1-(\ell+2) z^{2}\right) \phi^{(\ell+1,2)}(x)=\left(\frac{2 a}{s_{0}}\right)^{2} E_{n}^{(\ell+1)} \phi^{(\ell+1,2)}(x) .
$$

Equation (53) is again of Legendre type and its solutions in terms of eigenfunctions have the form:

$$
\phi_{n}^{(\ell+1,2)}(x)=Q_{\ell+1}^{n}\left(i \frac{A \sin \left(\frac{s_{0}}{2 a} x\right)-B \cos \left(\frac{s_{0}}{2 a} x\right)}{A \cos \left(\frac{s_{0}}{2 a} x\right)+B \sin \left(\frac{s_{0}}{2 a} x\right)}\right) .
$$

The energy spectrum is given by

$$
E_{n}^{(\ell+1)}=\left(\frac{s_{0}}{2 a}\right)^{2}\left(n^{2}-(\ell+1)^{2}\right) .
$$

Finally, one defines the annihilation, $A^{(\ell+1)}$, and creation, $\left(A^{(\ell+1)}\right)^{\dagger}$ operators, which transform the eigenvectors of $H^{(\ell+1,1)}$ into the eigenvectors of $H^{(\ell+1,2)}$ and reciprocally, respectively, as:

$$
\begin{gathered}
A^{(\ell+1)}=\partial_{x}+W^{(\ell+1)}(x)=\frac{i s_{0}}{2 a}\left(1-z^{2}\right) \partial_{z}-i(\ell+1) \frac{s_{0}}{2 a} z, \\
\left(A^{(\ell+1)}\right)^{\dagger}=-\partial_{x}+W^{(\ell+1)}(x)=-\frac{i s_{0}}{2 a}\left(1-z^{2}\right) \partial_{z}-i(\ell+1) \frac{s_{0}}{2 a} z .
\end{gathered}
$$

These creation and annihilation operators have been already constructed for the general formalism of SUSY potential partners in [1].

The relation between the Hamiltonian partners $H^{(\ell, 1)}$ and $H^{(\ell, 2)}$ for $\ell$ arbitrary are shown in Figure 5. For $\ell=0$, there is a unique Hamiltonian, which is $H_{\alpha}$. Now, the creation and annihilation operators in the $z$ variable give the recurrence identities for the associated Legendre functions.
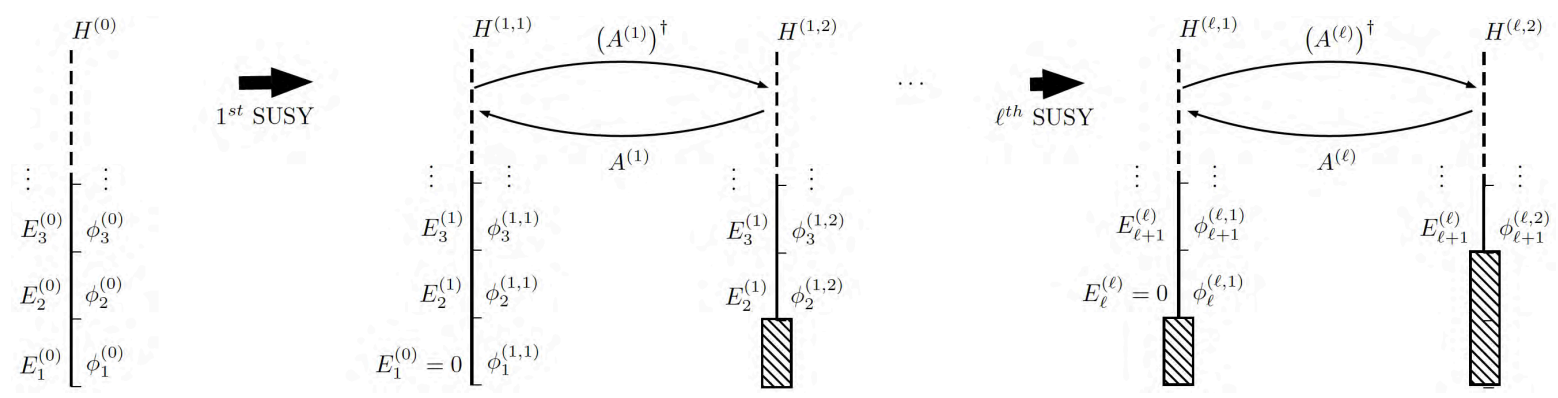

Figure 5. Energy scheme of different SUSY transformations up to order $\ell$.

\section{Conclusions and Outlook}

We have discussed the results of [15] for the self-adjoint extensions of the differential operator $H_{0}=-d^{2} / d x^{2}$ and gone beyond these results in the sense of addressing some cases not treated in [15]. Also, we have proposed a more detailed classification of the 
spectrum of these extensions in terms of the parameters that characterize each one of these extensions. We have seen that it is possible to classify these extensions in terms of other sets of variables with the sense of angles, which permits us to go beyond [15]. These self-adjoint extensions may have at most two negative eigenvalues, a ground state of zero energy and ground states with strictly positive energy.

In addition, in this paper, we have obtained analytically the form of the SUSY partners for the self-adjoint extensions of $H_{0}$ (that we denote as $H_{\alpha}$, where $\alpha$ includes the four real parameters that gives each of these extensions) with a ground state with positive energy. We have obtained all Hamiltonian partners of each of the $H_{\alpha}$ with positive spectrum to all orders, their energy levels and their eigenfunctions. At each step, we find two distinct Hamiltonian partners of $\ell$-th order. Creation and annihilation operators related the eigenfunctions for these two partners were also evaluated.

Although we have obtained the eigenfunctions for the whole sequence of SUSY partners of each of the $H_{\alpha}$, these eigenfunctions depend explicitly on the square root of the ground state energy of $H_{\alpha}$, which in most cases can be obtained by solving a transcendental equation. However, this transcendental equation looks rather intractable in a few cases. This situation poses some difficulties in obtaining the eigenvalues for some of the $H_{\alpha}$, although the explicit form of their eigenfunctions and of the eigenfunctions of their SUSY partners can always be given, as functions of the square root of the ground state energy of $H_{\alpha}$.

We have not obtained the SUSY partners for those extensions, $H_{\alpha}$, with a ground state with zero or negative energy. Here, we may also obtain a sequence of SUSY partners form each of the $H_{\alpha}$ in this class. Unlike the partners for extensions $H_{\alpha}$ with ground states with strictly positive energies, these partners may have a finite number of eigenvalues or even none, and the potential partners may show singularities. A classification of the partners for these exceptional extensions is left for a forthcoming paper.

Author Contributions: Writing original draft: M.G., J.H.M., L.M.N. and C.S.M.; Writing review editing: M.G., J.H.M., L.M.N. and C.S.M. All authors listed have made a substantial, direct, and intellectual contribution to the work. All authors have read and agreed to the published version of the manuscript.

Funding: This research was funded by Junta de Castilla y León and FEDER projects VA137G18 and BU229P18.

Data Availability Statement: Not applicable.

Conflicts of Interest: The authors declare no conflict of interest. The funders had no role in the design of the study; in the collection, analyses, or interpretation of data; in the writing of the manuscript, or in the decision to publish the resultss.

\section{Appendix A}

In this Appendix, we justify the correct choice of the wave functions for the bound states of the supersymmetric partners of each of the extensions $H_{\alpha}$ with strictly positive ground state energy. In Appendix A.1, we derive a general solution for these wave functions as a linear combination of the associated Legendre functions $P_{\ell}^{n}$ and $Q_{\ell}^{n}$ with argument $-\tan \left(\left(s_{0} x\right) /(2 a)\right)$. In Appendix A.2, we show that the component with $P_{\ell}^{n}$ should be discarded, since it does not meet the requirement of square integrability. On the other hand, the component with $Q_{\ell}^{n}$ should give the wave function as is square integrable, as proven in Appendix A.3.

\section{Appendix A.1}

Comments in these Appendices are valid for those self-adjoint extensions $H_{\alpha}$ with ground states with positive energy. For each of these extensions, the ground state energy is $E_{0}^{(0)}=\left(s_{0} /(2 a)\right)^{2}$, where $s_{0}$ depends on the chosen self-adjoint extensions and, therefore, on the values of the parameters. As we have seen, in terms of the auxiliary variable $s$, 
the spectrum is equally spaced in this case, so that all other energy values are $E_{m}^{(0)}=$ $\left(s_{0} /(2 a)\right)^{2} m^{2}$. For the ground state, the wave function is

$$
\phi_{m=1}^{(0)}(x)=A \cos \left(\frac{s_{0}}{2 a} x\right)+B \sin \left(\frac{s_{0}}{2 a} x\right) .
$$

The coefficients $A$ and $B$, as complex numbers, should have the same phase in order to have a real partner potential. To see it, let us write $A=C e^{i \varphi_{1}}$ and $B=D e^{i \varphi_{2}}$, with $C:=|A|$ and $D:=|B|$. Then, (A1) is

$$
\phi_{m=1}^{(0)}(x)=C e^{i \varphi_{1}} \cos \left(\frac{s_{0}}{2 a} x\right)+D e^{i \varphi_{2}} \sin \left(\frac{s_{0}}{2 a} x\right) .
$$

Using Definitions (42) and (43) for the potential partners of $\ell$-th order, we have for the first $\ell$-th partner:

$$
V^{(\ell+1,1)}=\frac{s_{0}^{2}(\ell+1)}{(2 a)^{2}}\left(-1+\ell\left(\frac{C e^{i \varphi_{1}} \sin \left(\frac{s_{0}}{2 a} x\right)-D e^{i \varphi_{2}} \cos \left(\frac{s_{0}}{2 a} x\right)}{C e^{i \varphi_{1}} \cos \left(\frac{s_{0}}{2 a} x\right)+D e^{i \varphi_{2}} \sin \left(\frac{s_{0}}{2 a} x\right)}\right)^{2}\right),
$$

for which the imaginary part is given by

$$
\begin{aligned}
\operatorname{Im}\left(V^{(\ell+1,1)}\right) & =\operatorname{Im}\left(\frac{s_{0}^{2}(\ell+1)}{(2 a)^{2}}\left(-1+\ell\left(\frac{A \sin \left(\frac{s_{0}}{22} x\right)-B \cos \left(\frac{s_{0}}{22} x\right)}{A \cos \left(\frac{s_{0}}{2 a} x\right)+B \sin \left(\frac{s_{0}}{2 a} x\right)}\right)^{2}\right)\right) \\
& =\frac{C D \ell(\ell+1) s_{0}^{2}\left((C-D)(C+D) \sin \left(\frac{s_{0} x}{a}\right)-2 C D \cos \left(\varphi_{1}-\varphi_{2}\right) \cos \left(\frac{s_{0} x}{a}\right)\right)}{a^{2}\left(2 C D \cos \left(\varphi_{1}-\varphi_{2}\right) \sin \left(\frac{s_{0} x}{a}\right)+(C-D)(C+D) \cos \left(\frac{s_{0} x}{a}\right)+C^{2}+D^{2}\right)^{2}} \sin \left(\varphi_{1}-\varphi_{2}\right),
\end{aligned}
$$

so that potential (A3) is real if $\sin \left(\varphi_{1}-\varphi_{2}\right)=0$, or equivalently, if $\varphi_{1}=n \pi+\varphi_{2}$. Thus, if $A$ and $B$ have the same phase as complex numbers, we have guaranteed that the potential partner $V^{(\ell+1,1)}$ is real. The same is valid for $V^{(\ell+1,2)}$. Thus, (A2) becomes:

$$
\phi_{m=1}^{(0)}(x)=C e^{i \varphi} \cos \left(\frac{s_{0}}{2 a} x\right)+D e^{i \varphi} \sin \left(\frac{s_{0}}{2 a} x\right) .
$$

This ground state is not yet normalized. Its normalization gives

$$
\int_{-a}^{a} d x \phi_{m=1}^{(0)}(x)\left(\phi_{m=1}^{(0)}(x)\right)^{*}=1 \quad \Longrightarrow \quad C^{2}+D^{2}=1 \quad \Longrightarrow \quad C=\cos \delta, D=\sin \delta .
$$

Finally, the ground state wave function has the form:

$$
\phi_{m=1}^{(0)}(x)=e^{i \varphi} \cos \left(\frac{s_{0}}{2 a} x+\delta\right) .
$$

Let us recall that our goal is to show that the solution of the Schrödinger equation with component $Q_{\ell}^{n}$ is square integrable and the solution with $P_{\ell}^{n}$ is not. To begin with, let us define a new independent variable using the shift $x=y-2 a \delta / s_{0}$. The ground state has now the form,

$$
\phi_{m=1}^{(0)}=e^{i \varphi} \cos \left(\frac{s_{0}}{2 a} y\right) .
$$

With this notation, the wave function of the second partner of $\ell$-th order is

$$
\phi_{m}^{(\ell, 2)}=C_{1} P_{\ell}^{n}\left(-i \tan \left(\frac{s_{0} y}{2 a}\right)\right)+C_{2} Q_{\ell}^{n}\left(-i \tan \left(\frac{s_{0} y}{2 a}\right)\right) .
$$

Next, we shall analyze the square integrability of each of the components in (A9). 


\section{Appendix A.2. Trigonometric Expansion of $P_{\ell}^{n}\left(-i \tan \left(\frac{s_{0} y}{2 a}\right)\right)$}

Let us use the change of variable $z=-i \tan \left(\frac{s_{0} y}{2 a}\right)$ and consider the hypergeometric form of the associated Legendre functions with argument $z$ [38]:

$$
\begin{aligned}
P_{\ell}^{n}(z) & =\frac{1}{\ell !}\left(-\frac{1}{2}\right)^{\ell}\left(\frac{1+z}{1-z}\right)^{n / 2}(1-z)^{\ell} \frac{\Gamma(2 \ell+1)_{2} F_{1}\left(-\ell, n-\ell ;-2 \ell ;-\frac{2}{z-1}\right)}{\Gamma(\ell-n+1)} \\
& =\frac{1}{\ell !}\left(-\frac{1}{2}\right)^{\ell}\left(\frac{1+z}{1-z}\right)^{n / 2}(1-z)^{\ell} \sum_{j=0}^{\ell} \frac{\left((2 \ell-j) !(-\ell)_{j}\right)}{j ! \Gamma(-j+\ell-n+1)}\left(\frac{2}{1-z}\right)^{j} \\
& =\frac{\left(-\frac{1}{2}\right)^{\ell} \Gamma(2 \ell+1)}{\ell ! \Gamma(\ell-n+1)} \frac{e^{-\frac{i s_{0}(n-\ell)}{2 a} y}}{\cos ^{\ell}\left(\frac{s_{0} y}{2 a}\right)} \sum_{j=0}^{\ell} \frac{(-1)^{j} \Gamma(\ell+1)(2 \ell-j) !}{j ! \Gamma(-j+\ell+1) \Gamma(-j+\ell-n+1)}\left(2 e^{-\frac{i s_{0}}{2 a} y} \cos \left(\frac{s_{0} y}{2 a}\right)\right)^{j} \\
& =\sum_{j=0}^{\ell} \frac{(-1)^{j+\ell} 2^{j-\ell} \Gamma(\ell+1)(2 \ell-j) !}{j ! ! \Gamma(-j+\ell+1) \Gamma(-j+\ell-n+1)} e^{-\frac{i \delta_{0} y(j-\ell+n)}{2 a}} \cos ^{j-\ell}\left(\frac{s_{0} y}{2 a}\right) .
\end{aligned}
$$

Due to the presence of negative powers of the cosine in (A10), the resulting wave function is not square integrable and, therefore, not acceptable as a wave function of a bound state.

Appendix A.3. Trigonometric Expansion of $Q_{\ell}^{n}\left(-i \tan \left(\frac{s_{0} y}{2 a}\right)\right)$

Similarly, we can express $Q_{\ell}^{n}(z)$ in terms of a hypergeometric function [38] as:

$$
Q_{\ell}^{n}(z)=\frac{1}{\sqrt{\pi}} 2^{-\ell-1}(-1)^{\ell+n+1}(z-1)^{-\ell-1} \Gamma\left(-\ell-\frac{1}{2}\right)\left(\frac{z+1}{z-1}\right)^{n / 2}(\ell+n) !{ }_{2} F_{1}\left(\ell+1, \ell+n+1 ; 2(\ell+1) ;-\frac{2}{z-1}\right) .
$$

Then, let us perform again the change of variables given by $z=-i \tan \left(\frac{s_{0} y}{2 a}\right)$, so as to obtain:

$$
\begin{aligned}
Q_{\ell}^{n}\left(-i \tan \left(\frac{s_{0} y}{2 a}\right)\right)= & \frac{1}{\sqrt{\pi}} 2^{-\ell-1}(-1)^{n} \Gamma\left(-\ell-\frac{1}{2}\right) \Gamma(\ell+n+1) e^{-i \frac{s_{0}}{2 a} y(\ell+n+1)} \cos ^{\ell+1}\left(\frac{s_{0} y}{2 a}\right) \\
& \times{ }_{2} F_{1}\left(\ell+1, \ell+n+1 ; 2(\ell+1) ; 2 e^{-i \frac{s_{0} y}{2 a}} \cos \left(\frac{s_{0} y}{2 a}\right)\right) .
\end{aligned}
$$

If again, we perform a series expansion around $z=0$ we obtain the following power series in terms of positive powers of cosines:

$$
Q_{\ell}^{n}\left(-i \tan \left(\frac{s_{0} y}{2 a}\right)\right)=\frac{1}{2}(-1)^{-\ell+n+1} e^{-i \frac{s_{0}}{2 a} n y} \Gamma(n-\ell) \Gamma(\ell+n+1) \sum_{j=\ell+1}^{n} \frac{(-1)^{j} \Gamma(j) 2^{j} e^{i j} \frac{s_{0}}{2 a} y}{\Gamma(j-\ell) \Gamma(j+\ell+1) \Gamma(-j+n+1)} \cos ^{j}\left(\frac{s_{0}}{2 a} y\right) .
$$

This solution is acceptable as is square integrable.

\section{References}

1. Fernández, D.J. Supersymmetric quantum mechanics. AIP Conf. Proc. 2010, 1287, 3-36.

2. Infeld, L.; Hull, T.E. The factorization method. Rev. Mod. Phys. 1951, 23, 21-68. [CrossRef]

3. Lahiri, A.; Roy, P.K.; Bagchi, B. Supersymmetry in quantum mechanics. Int. J. Mod. Phys. A 1990, 5, 1383-1456. [CrossRef]

4. Roy, B.; Roy, P.; Roychoudhury, R. On the solution of quantum eigenvalue problems. A supersymmetric point of view. Fortschr. Phys. 1991, 39, 211-258. [CrossRef]

5. Bagchi, B. Superymmetry in Quantum and Classical Mechanics; Chapman and Hall: Boca Raton, FL, USA, 2001.

6. Cooper, F.; Khare, A.; Sukhatme, U. Supersymmetry and Quantum Mechanics. Phys. Rep. 1995, 251, 267-385. [CrossRef]

7. Díaz, J.I.; Negro, J.; Nieto, L.M.; Rosas-Ortiz, O. The supersymmetric modified Pöschl-Teller and delta-well potential. J. Phys. A Math. Gen. 1999, 32, 8447-8460. [CrossRef]

8. Mielnik, B.; Nieto, L.M.; Rosas-Ortiz, O. The finite difference algorithm for higher order supersymmetry. Phys. Lett. A 2000, 269, 70-78. [CrossRef]

9. Fernández, C.D.J.; Negro, J.; Nieto, L.M. Regularized Scarf potentials: Energy band structure and supersymmetry. J. Phys. A Math. Gen. 2004, 37, 6987-7001. 
10. Ioffe, M.V.; Negro, J.; Nieto, L.M.; Nishiniandze, D. New two-dimensional integrable quantum models from SUSY intertwining. J. Phys. A Math. Gen. 2006, 39, 9297-9308. [CrossRef]

11. Correa, F.; Nieto, L.M.; Plyushchay, M.S. Hidden nonlinear supersymmetry of finite-gap Lamé equation. Phys. Lett. B 2007, 644, 94-98. [CrossRef]

12. Ganguly, A.; Nieto, L.M. Shape-invariant quantum Hamiltonian with position-dependent effective mass through second-order supersymmetry. J. Phys. A Math. Gen. 2007, 49, 7265-7281. [CrossRef]

13. Correa, V.; Jakubsk, V.; Nieto, L.M.; Plyushchay, M.S. Self-isospectrality, special supersymmetry, and their effect on the band structure. Phys. Rev. Lett. 2008, 101, 030403. [CrossRef]

14. Fernández, C.D.J.; Gadella, M.; Nieto, L.M. Supersymmetry Transformations for Delta Potentials. SIGMA 2011, 7, 029. [CrossRef]

15. Bonneau, G.; Faraut, J.; Valent, G. Self adjoint extensions of operators and the teaching of Quantum Mechanics. Am. J. Phys. 2001, 69, 322-331. [CrossRef]

16. Albeverio, S.; Fassari, S.; Rinaldi, F. The Hamiltonian of the harmonic oscillator with an attractive $\delta^{\prime}$-interaction centred at the origin as approximated by the one with a triple of attractive delta-interactions. J. Phys. A Math. Theor. 2016, 49, 025302. [CrossRef]

17. Zolotaryuk, A.V.; Tsironis, G.P.; Zolotaryuk, Y. Point Interactions With Bias Potentials. Front. Phys. 2019, 7, 87. [CrossRef]

18. Zolotaryuk, A.V. A phenomenon of splitting resonant-tunneling one-point interactions. Ann. Phys. 2018, 396, 479-494. [CrossRef]

19. Zolotaryuk, A.V.; Zolotaryuk, Y. A zero-thickness limit of multilayer structures: A resonant-tunnelling $\delta^{\prime}$-potential. J. Phys. A Math. Theor. 2015, 48, 035302. [CrossRef]

20. Golovaty, Y.D.; Hryniv, R.O. On norm resolvent convergence of Schrödinger operators with $\delta^{\prime}$-like potentials. J. Phys. A Math. Theor. 2010, 43, 155204. [CrossRef]

21. Erman, F.; Uncu, H. Green's function formulation of multiple nonlinear Dirac delta-function potential in one dimension. Phys. Lett. A 2020, 384, 126227. [CrossRef]

22. Donaire, M.; Muñoz-Castañeda, J.M.; Nieto, L.M.; Tello-Fraile, M. Field Fluctuations and Casimir Energy of 1D-Fermions. Symmetry 2019, 11, 643. [CrossRef]

23. Gadella, M.; Mateos-Guilarte, J.M.; Muñoz-Castañeda, J.M.; Nieto, L.M. Two-point one-dimensional $\delta(x)-\delta^{\prime}(x)$ interactions: Non-abelian addition law and decoupling limit. J. Phys. A Math. Theor. 2016, 49, 015204. [CrossRef]

24. Gadella, M.; Mateos-Guilarte, J.M.; Muñoz-Castañeda, J.M.; Nieto, L.M.; Santamaría-Sanz, L. Band spectra of periodic hybrid $\delta$ - $\delta^{\prime}$ structures. Eur. Phys. J. Plus 2020, 135, 786. [CrossRef]

25. Reed, M.; Simon, B. Fourier Analysis. Self Adjointness; Academic Press: New York, NY, USA, 1975.

26. Fassari, S.; Gadella, M.; Glasser, M.L.; Nieto, L.M. Spectroscopy of a one-dimensional V-shaped quantum well with a point impurity. Ann. Phys. 2018, 389, 48-62. [CrossRef]

27. Kurasov, P. Distribution theory for discontinuous test functions and differential operators with generalized coefficients. J. Math. Ann. Appl. 1996, 201, 297-323. [CrossRef]

28. Charro, M.E.; Glasser, M.L.; Nieto, L.M. Dirac Green function for $\delta$ potentials. EPL 2017, 120, 30006. [CrossRef]

29. Fassari, S.; Gadella, M.; Nieto, L.M.; Rinaldi, F. On the spectrum of the one-dimensional Schrödinger Hamiltonian perturbed by an attractive Gaussian potential. Acta Politech. 2017, 57, 385-390. [CrossRef]

30. Muñoz-Castañeda, J.M.; Nieto, L.M.; Romaniega, C. Hyperspherical $\delta-\delta^{\prime}$ potentials. Ann. Phys. 2019, 400, 246-261. [CrossRef]

31. Albeverio, S.; Fassari, S.; Gadella, M.; Nieto, L.M.; Rinaldi, F. The Birman-Schwinger Operator for a Parabolic Quantum Well in a Zero-Thickness Layer in the Presence of a Two-Dimensional Attractive Gaussian Impurity. Front. Phys. 2019, 7, 102. [CrossRef]

32. Gadella, M.; García-Ferrero, M.A.; González-Martín, S.; Maldonado-Villamizar, F.H. The infinite square well with a point interaction: A discussion on the different parameterizations. Int. J. Theor. Phys. 2013, 53, 1614-1627. [CrossRef]

33. Gadella, M.; Glasser, M.L.; Nieto, L.M. The infinite square well with a singular perturbation. Int. J. Theor. Phys. 2011, 50, 2191-2200. [CrossRef]

34. Naimark, M.A. Linear Differential Operators; Dover: New York, NY, USA, 2014.

35. Gadella, M.; Glasser, M.L.; Nieto, L.M. One Dimensional Models with a Singular Potential of the Type $-\alpha \delta(x)+\beta \delta^{\prime}(x)$. Int. J. Theor. Phys. 2011, 50, 2144-2152. [CrossRef]

36. Reed, M.; Simon, B. Functional Analysis; Academic Press: New York, NY, USA, 1972.

37. Bachman, G.; Narici, L. Functional Analysis; Dover: New York, NY, USA, 2012.

38. Olver, F.W.J.; Lozier, D.W.; Boisvert, R.F.; Clark, C.W. NIST Handbook of Mathematical Functions, 1st ed.; Cambridge University Press: Cambridge, UK, 2010. Available online: https:/ / dlmf.nist.gov/14.3 (accessed on 6 December 2020). 